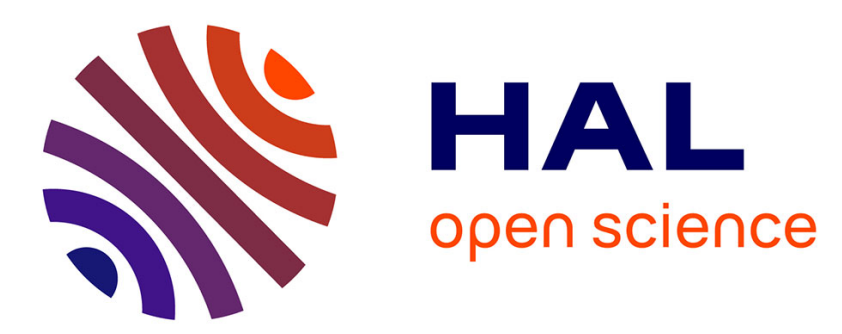

\title{
Advanced thermo-mechanical characterization of organic materials by piezoresistive organic resonators
}

Damien Thuau, Cédric Ayela, Etienne Lemaire, Stephen Heinrich, Philippe Poulin, Isabelle Dufour

\section{- To cite this version:}

Damien Thuau, Cédric Ayela, Etienne Lemaire, Stephen Heinrich, Philippe Poulin, et al.. Advanced thermo-mechanical characterization of organic materials by piezoresistive organic resonators. Materials Horizons, 2015, 2, pp.106-112. 10.1039/C4MH00165F . hal-01073816

\section{HAL Id: hal-01073816 https://hal.science/hal-01073816}

Submitted on 16 Dec 2014

HAL is a multi-disciplinary open access archive for the deposit and dissemination of scientific research documents, whether they are published or not. The documents may come from teaching and research institutions in France or abroad, or from public or private research centers.
L'archive ouverte pluridisciplinaire HAL, est destinée au dépôt et à la diffusion de documents scientifiques de niveau recherche, publiés ou non, émanant des établissements d'enseignement et de recherche français ou étrangers, des laboratoires publics ou privés. 


\title{
Advanced thermo-mechanical characterization of organic materials by piezoresistive organic resonators
}

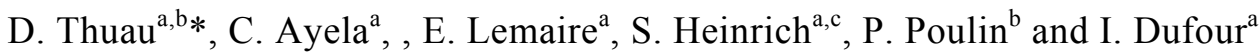

We present the piezoresistive transduction of an all-organic microelectromechanical (MEMS) resonant sensor fabricated through a low-cost and highly versatile process. The MEMS resonator consists of a U-shaped cantilever beam resonator made of a thin layer of a piezoresistive nanocomposite (SU/8 epoxy resin filled with industrially produced carbon nanotubes, or CNTs) deposited on flexible substrates such as polyethylene terephthalate (PET), polyethylene naphthalate (PEN) and paper. The structures have been fabricated using a commercially available vinyl cutting machine. External piezoelectric actuation has been used to drive the devices into resonance while integrated piezoresistive transduction has been chosen as the resonance sensing approach. The achieved measurements validate the concept of dynamic piezoresistive-transduced organic MEMS. Sensitivity to temperature compares with that of state-of-the-art inorganic temperature sensors, thus confirming the high accuracy level of the new resonators. As an example of a sensing application, the present MEMS are employed as microdynamical mechanical analyzers enabling the rapid, low-cost and accurate characterization of the viscoelastic properties of organic materials.

\begin{abstract}
A Introduction
The fast growing market that represents organic electronics is of huge interest in the MEMS field. ${ }^{1-2}$ Organic electronics offers two major advantages in comparison to classical siliconbased electronics: (1) it enables the design of devices on flexible substrates and offers a wide range of new applications in terms of flexibility; (2) it allows the production of electronics applications with fabrication techniques whose investments and costs are much lower than in the silicon industry. Although the performance of silicon MEMS is often considered as higher than organic MEMS, the cost/performance ratio makes these organic electronics devices an extremely attractive alternative option for many applications.

A key parameter for the development of organic electronicsbased microsystems is therefore the expertise and control of appropriate fabrication processes. To date, two main techniques are used to deposit organic materials: (1) evaporation and spincoating and (2) printing techniques (spray, ink jet, screen printing). Thus, organic materials have generally been patterned using standard technology processes from the semiconductor industry. However, significant levels of attention are now focused on novel fabrication techniques to achieve the optimal cost/performance ratio required to launch this emerging market. The fabrication technology proposed and realized in this work for the development of an organic piezoresistive resonator addresses this issue. In fact, it enables the fabrication of polymer MEMS without cleanroom micro-fabrication facilities and should lead to broaden the use of resonator in the same spirit as PDMS microfluidic or 3D printing. Here, a commercially available vinyl cutting machine ${ }^{3}$ has been used to design organic MEMS resonators at an extremely low cost and
\end{abstract}

rapid production rate with no sacrifice in performance. Moreover, by using the proposed fabrication technology the manufacturing cost of an organic electronic device is drastically reduced compared to an inorganic device. Thus, the role of materials in the overall cost of manufacturing, as presented here, can be estimated to represent a much larger portion than in the semiconductor industry, in which it represents only a small fraction of the cost. This addresses the strategic challenge that represents materials design (substrates and deposited layers with specific electrical, chemical and mechanical properties) for the development of organic electronics. In this sense, flexible substrates such as paper and plastic have been chosen in this work to further reduce the cost of the final sensor. Functionality is provided by carbon nanotubes that are produced on an industrial scale and are now available at a cost estimated to be below $50 \mathrm{USD} / \mathrm{kg}$. Organic/inorganic hybrid materials and nanocomposite materials have been shown to display performances that compare or exceed that of an inorganic semiconductor when used as a transduction element or sensing layer in MEMS devices. ${ }^{4-7}$ In particular, CNT- based piezoresistive nanocomposites exhibit giant gauge factors due to the intrinsic gauge factor of an individual single-walled nanotube (SWNT) and to changes of the contact resistances in response to mechanical deformations. ${ }^{8}$ Depending on the applied stimuli, their gauge factors have been found to range from unity to several hundred. This type of strain sensor exhibiting outstanding static and low-frequency dynamic response shows promise for implementation for structural health/integrity monitoring tasks such as dynamic contact detection or monitoring of impact events. ${ }^{7}$ In addition to outstanding strain sensing capabilities, piezoresistive 
nanocomposites are viewed as powerful transducers for realtime MEMS chemical and bio-sensing applications.

Due to its relatively simple geometry and ease of fabrication, the most common platform for MEMS sensing applications is the cantilever beam. Here, U-shaped cantilevers have been chosen for the resonator geometry in order to concentrate the strain on the piezoresistor. Cantilever-based MEMS can be operated either in a static bending regime (where the cantilever bends due to mechanical strain) or in a dynamic mode.In the latter case, the phenomenon to be sensed may be revealed with excellent resolution by a shift in the resonant frequency of the cantilever caused by, e.g., sorbed mass and/or environmentally induced changes in material properties. Sensing based on resonant frequency shift provides more precision than sensing in the static regime since the effect of noise in a direct voltage or current measurement is clearly attenuated in frequency measurements. To date, the piezoresistive responses of organic MEMS have only been reported in the static bending configuration. ${ }^{6-10}$ This provides a major motivation for the primary objective of the present work: to present the first piezoresistively transduced organic cantilevers functioning in a low-frequency dynamic configuration. The reported sensors have been fabricated using an innovative, low-cost and environmentally friendly fabrication process which employs commercially available materials. Electromechanical characterization of the organic cantilevers as a function of temperature has been performed with the aim of validating this new concept. Finally, the electromechanical characterization of the organic MEMS has been used to analyze the viscoelastic mechanical properties of particular organic materials.

\section{B Materials and methods}

The micromachining method of our approach does not require the high-cost semiconductor manufacturing equipment used for silicon microfabrication and therefore drastically reduces the cost of the MEMS devices in comparison with those of the silicon industry in which 65-nm technology has required a multi-billion dollar investment. Here, an extremely low-cost fabrication process has been developed for the fabrication of organic MEMS. This process requires a spin coater to deposit the material and, as noted earlier, a vinyl cutting machine to pattern the structure. Furthermore, the main microfabrication concerns of deposited layer homogeneity and the resolution of the patterned structure are addressed by combining spin- or spray-coating, yielding a deposited layer thickness ranging from $100 \mathrm{~nm}$ to hundreds of microns, with the vinyl cutting machine (resolution of $5 \mu \mathrm{m}$ ). ${ }^{3}$ For comparison purposes, the cost of the equipment for this fabrication technique has been estimated to be less than 4,000 USD. Consequently, the proposed fabrication technique can be applied to a wide range of polymers leading to a straightforward development of organic electronics devices and microsystems. Figure 1a illustrates the fabrication process flow of the organic resonator. This fabrication process replaces UV photolithography as the easiest way to pattern polymer MEMS and has been demonstrated to be the most straightforward method to pattern organic MEMS. ${ }^{11}$.
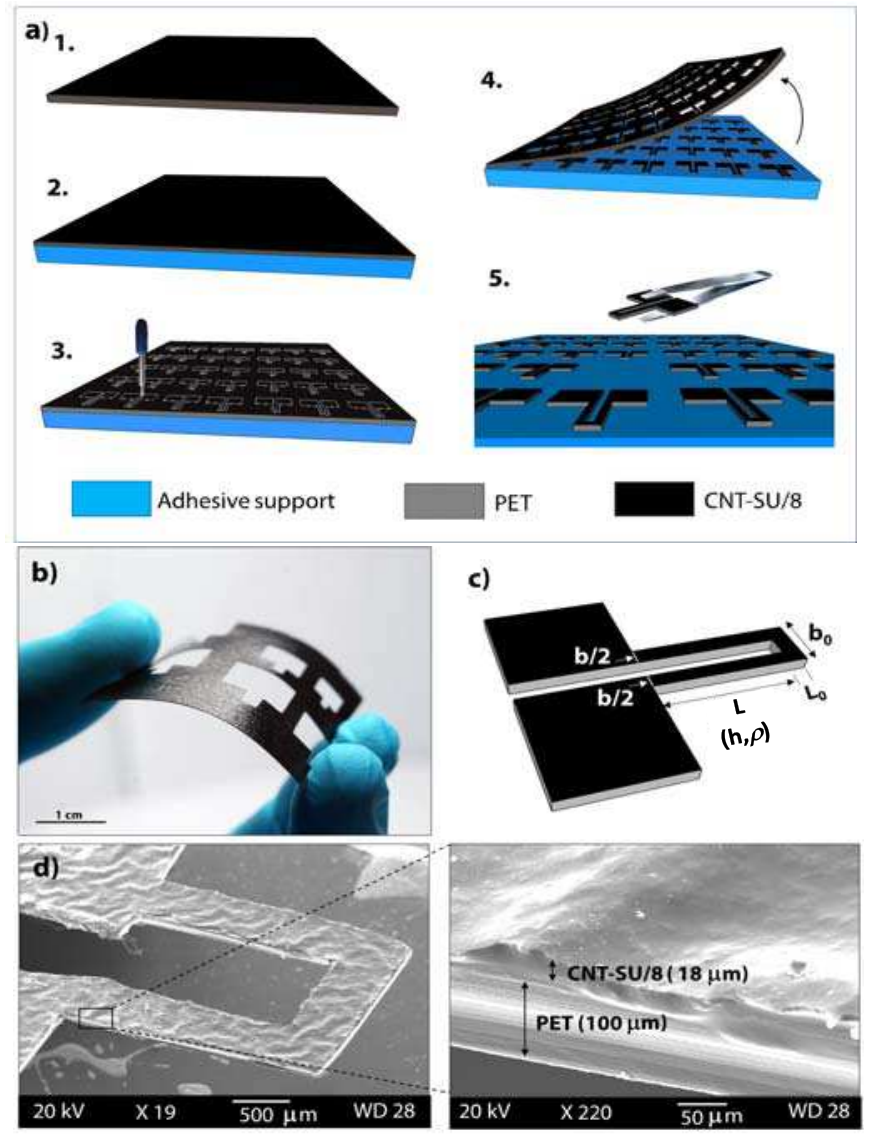

Figure 1a) Fabrication process flow for the U-shaped organic resonator, 1b) Optical image showing the flexibility of the bimorph nanocomposite + PET sheet after patterning, 1c) Schematic of the U-shaped cantilever (with h, the thickness and $\rho$, the density) and 1d) SEM images of the U-shaped organic $M E M S$

The first step involves the preparation of the piezoresistive nanocomposite. CNT-based Graphistrength Epoxy Master batch pellets from Arkema have been used. The pellets consist of $25 \mathrm{wt} \%$ of multi-walled nanotubes (MWNTs) made via catalytic chemical vapor deposition (CCVD) and dispersed in an epoxy-type matrix. The nanocomposite was prepared by mixing the SU-8 epoxy photoresist with the pellets using a high shear mixer (Silverson L4RT) at $5000 \mathrm{rpm}$ for $60 \mathrm{~min}$ in an ice bath. Subsequently, the U-shaped piezoresistive organic cantilevers were fabricated in a low-cost, two-step process. The aforementioned solution was spin-coated on a $100-\mu \mathrm{m}$ thick sheet of polyethylene terephthalate (PET) and soft-baked at $95^{\circ} \mathrm{C}$ for $5 \mathrm{~min}$. The thin film was then cross-linked by exposure to UV light combined with a post-exposure bake step at $65^{\circ} \mathrm{C}$ for $1 \mathrm{~min}$ and $120^{\circ} \mathrm{C}$ for $10 \mathrm{~min}$ followed by a final hard bake of $150^{\circ} \mathrm{C}$ for $15 \mathrm{~min}$ to release internal stress. Afterwards, the resonators were patterned by simply using the vinyl cutting machine (Graphtec Craft ROBO Pro CE 5000-40). Note that the thickness of the layer to be patterned should be 
thick enough (>10um) to be easily manipulated during fabrication and thinner than the limitation of the vinyl cutting plotter (250um). The final dimensions of the PET-based structures were $2800 \mu \mathrm{m}$ long, $1600 \mu \mathrm{m}$ wide and $118 \mu \mathrm{m}$ thick. Figure 1b shows a nanocomposite/PET bimorph flexible thin film after MEMS device patterning while Figure 1c represents a schematic of the U-shaped MEMS cantilever. SEM images of the fabricated PET-CNT-SU/8 resonator are shown in Figure 1d.

\section{Results and discussion}

\section{Piezoresistive transduction of MEMS resonator}

The piezoresistive behavior of a CNT network within an insulating matrix involves a complicated interplay among different mechanisms, including the resistance change of the matrix due to dimensional changes, tunneling resistance, resistance of the intertube contacts and intrinsic piezoresistivity of the nanotubes. ${ }^{12}$ Several numerical studies have been performed with the aim of theoretically describing the influence of the CNTs and polymer parameters on the piezoresisitive effect in nanocomposites (e.g., ${ }^{13,14}$ ). A general observation made in these studies is the enhancement of the sensitivity of a piezoresisitive nanocomposite for samples containing a CNT wt $\%$ concentration close to the percolation threshold. This phenomenon was also reported in several experimental studies; for instance, in the case of a similar material as the ones used in this work, namely an epoxy resin filled with carbon-based particles. ${ }^{5,6}$ A detailed electrical characterization as well as an analytical modeling of the piezoresistive behavior of the CNT$\mathrm{SU} / 8$ composite has been reported in a previous work. ${ }^{6}$ Accordingly, a nanocomposite containing $2 \mathrm{wt} \%$ of CNT concentration has been chosen to pattern the piezoresistive transducers with initial resistances of $6.1 \mathrm{M} \Omega$ on the resonator of the present study.

The fabricated devices were successfully driven into resonance by an external piezoelectric buzzer while optical and piezoresistive transductions were measured in order to ensure the accuracy of the piezoresistive transduction. Furthermore, finite element model (FEM) simulations using COMSOL were performed for the case of a homogeneous U-shaped resonator with the same dimensions and mechanical properties (measured with Dynamic Mechanical Analysis, (DMA)) of the CNT-SU/8 - PET fabricated device in order to investigate the structure's resonant frequency and quality factor. Finite element simulations together with the experimentally measured frequency response are plotted in Figure 2a while the inset depicts a snapshot of the model's resonant response. The simulations were performed by specifying the cantilever material's properties as density $\rho=1380 \mathrm{~kg} \cdot \mathrm{m}^{-3}$ and storage and loss moduli $E^{\prime}=4.43 \mathrm{GPa}$ and $E "=130 \mathrm{MPa}$, respectively. Using these input parameters, the resonant frequency and quality factor (due to viscoelastic material losses) of the first out-ofplane flexural mode were calculated to be $3493 \mathrm{~Hz}$ and 22, respectively, showing good agreement with the measured values (i.e., errors less than 3\% in resonant frequency and 5\% in quality factor as detailed in what follows).

The vibration amplitudes at resonance were detected with a laser vibrometer (Polytec MSA 500) whereas the electromechanical measurements were recorded with a network analyzer (Agilent E5061B). The devices were tested in a half
Wheatstone bridge configuration without using any amplification. All measurements were performed in air at atmospheric pressure. The resonant frequencies of the first outof-plane flexural mode were measured experimentally to be 3602 and $3597 \mathrm{~Hz}$ using the piezoresistive optical and transduction methods, respectively, thus showing excellent dynamic electromechanical transduction of the piezoresistive nanocomposite. Quality factor $(Q)$, defined as the ratio of the resonant frequency to the bandwidth associated with a $3 \mathrm{~dB}$ transmission magnitude drop, has been calculated from the data to be 23 and 21 for optical and piezoresistive transduction, respectively.

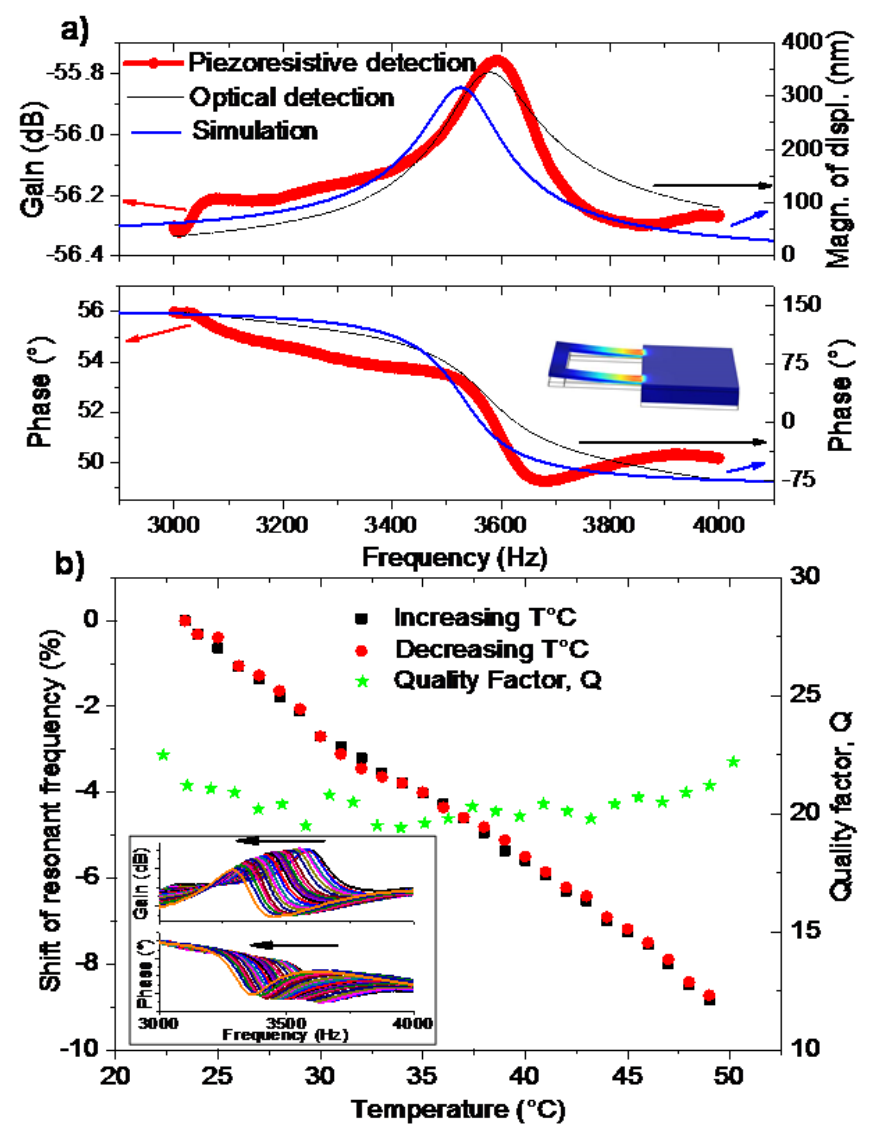

Figure 2a) Comparison between the frequency response of FEM simulations and experimental data based on optical and piezoresistive detection through the 1 st flexural mode of resonance; inset: snapshot of the model's resonant response 2b) Shift of the resonant frequency and quality factor as a function of temperature obtained from electrical measurement of the piezoresistance; inset: Measured frequency response curves for different temperatures where the arrows show the direction of the shift of the resonant frequency with increasing temperature

\section{Temperature sensor}

Additionally, the electromechanical responses of organic Ushaped microcantilever resonators as a function of temperature have been measured in order to validate the ability of the present piezoresistive transduction scheme integrated onto a PET resonator to be able to monitor accurately thermal effects occurring in the resonator. The measured relative changes of 
resonant frequency $\left(\Delta f_{r} / f_{r}\right)$ as well as quality factor versus temperature are plotted in Figure 2b, while the evolution of the resonant frequency of the first out-of-plane flexural mode of the $\mathrm{U}$-shaped cantilever resonator in the temperature range $23^{\circ} \mathrm{C}$ to $49^{\circ} \mathrm{C}$ is shown in the inset of Figure $2 \mathrm{~b}$. The resonant frequencies are observed to drop $\sim 9 \%$ from $3597 \mathrm{~Hz}$ to 3277 $\mathrm{Hz}$ when the temperature increases from $23^{\circ} \mathrm{C}$ to $49^{\circ} \mathrm{C}$. Moreover, the piezoresistive responses to temperature change show an excellent linearity with a correlation coefficient (R) of 0.9975 in this temperature range. The sensitivity of the PETbased resonator, as measured by the thermal coefficient of frequency (TCF) of the MEMS, has been determined from linear regression to be $-0.33 \% /{ }^{\circ} \mathrm{C}$ in the range $23-49^{\circ} \mathrm{C}$. The limit of detection (LOD) of the fabricated MEMS resonator has been estimated by calculating the standard deviation of the resonant frequency of 100 points at a given constant temperature and humidity level. The LOD could then be determined by taking three times the standard deviation and dividing it by the sensor' sensitivity. Using this approach the LOD of the proposed sensor was estimated to be $0.112^{\circ} \mathrm{C}$ Moreover, the quality factor has been calculated at different temperatures without exhibit significant changes. An average value of $Q=21 \pm 1$ has been measured. It is noted that standard commercial platinum temperature sensors (Pt100) have a relative sensitivity $(\Delta R / R / \Delta T$ with $R$ being the resistance) of $0.385 \% /{ }^{\circ} \mathrm{C}$ and a limit of detection in the range $0.143^{\circ} \mathrm{C}$ to $0.850^{\circ} \mathrm{C}$ depending on their characteristics. In comparison, our fabricated MEMS resonators exhibit sensitivity on the same order of magnitude and a better (lower) limit of detection $\left(0.112^{\circ} \mathrm{C}\right)$. This sensitivity to temperature confirms the high efficiency of the proposed resonators which is one order of magnitude higher than the best organic/inorganic bimorph materials reported to date and can compare with those based on the state-of-the-art inorganic materials. ${ }^{15-18}$ In addition, the stability of our sensors to humidity has been investigated where a shift in resonant frequency lower than $0.7 \%$ has been observed in the relative humidity range of 10 to $90 \% \mathrm{RH}$.

In order to obtain further insight into the temperaturedependence of the electromechanical properties of the MEMS device, the relative changes in resistance of CNT-SU/8 thin film as well as in the U-shaped cantilever in the static mode have been recorded as a function of temperature as shown in Figure $3 \mathrm{a}$. The resistance of CNT-SU/8 thin film decreases with increasing temperature, indicating a negative temperature coefficient. This behaviour can be explained by a thermal fluctuation-induced tunnelling conduction. ${ }^{19-21}$ Also shown in Fig. $3 \mathrm{a}$ is the dependence of the relative change in resonant frequency on the temperature of the U-shaped cantilever operated in the dynamic mode. The numerical values of the thermal sensitivities of the thin film and the U-shaped cantilever in static and dynamic modes have been measured to be $-863,-976$ and $-3300 \mathrm{ppm} /{ }^{\circ} \mathrm{C}$, respectively, demonstrating an improved thermal monitoring sensitivity of the MEMS when operating in the dynamic mode.

The real-time responses to temperature of the U-shaped cantilever used in static and dynamic mode have also been measured as shown in Figure $3 \mathrm{~b}$. One can observe the instantaneous response of the device when used in the dynamic mode whereas a long delay in the resistance change response to temperature can be seen, due to the viscoelastic relaxation of the material, when used in the static mode. Therefore, the integrated piezoresistive transduction in the dynamic mode clearly appears to be the method with optimal efficiency for sensing thermal events.
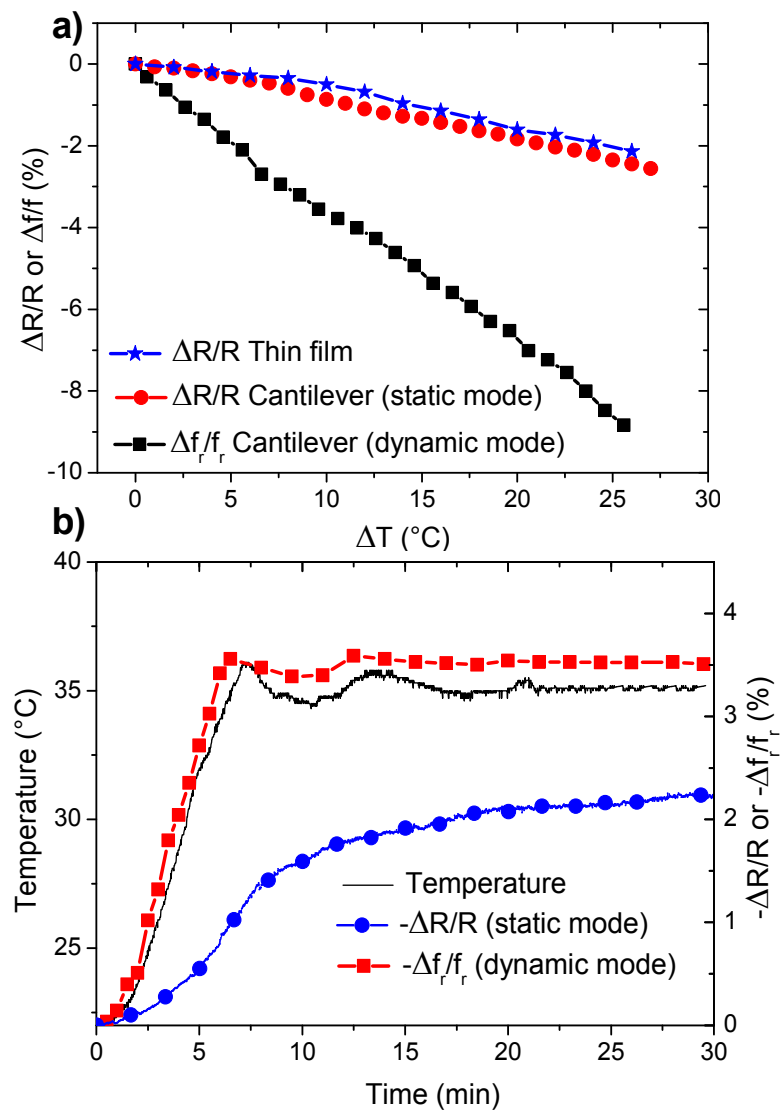

Figure 3a) Relative change in resistance (static mode) or resonant frequency (dynamic mode) for thin film and U-shaped cantilever as a function of $\Delta T$ with an initial temperature of $23^{\circ} \mathrm{C}$; $3 \mathrm{~b}$ ) Temperature control and real time responses of the $M E M S$ in static and dynamic mode

\section{Micro-dynamical mechanical analyser}

The performances of the proposed organic MEMS with integrated transduction scheme make these devices excellent candidates as mechanical platform to characterize accurately material properties. Indeed, by following the shift in resonant frequency and quality factor of the resonators as a function of temperature, one may determine the mechanical properties of the resonator material in a similar manner as in dynamic mechanical analysis (DMA) measurements. ${ }^{22-24}$ The use of MEMS as an analytical tool for thermomechanical characterization of polymer materials has recently been reported by S. Bose et al. using the resonant frequency and quality factor of microstrings measured by external optical transduction. The devices were made of silicon nitride $\left(\mathrm{SiN}_{\mathrm{x}}\right)$ fabricated using an expensive inorganic fabrication processes. Devices of this type enable the observation of the glass transition temperature $\left(T_{g}\right)$ of polymers via a relative variation of the Young's modulus. ${ }^{22}$ The present organic MEMS, in addition to being more simply fabricated, allows for more detailed quantitative measurements. One primary advantage of the device proposed here in comparison with the one reported by $\mathrm{S}$. Bose et al. ${ }^{22}$ is its integrated piezoresistive transduction scheme, which offers potential integration on multiple supports. Moreover, instead of assuming two constant values of Young's modulus before and after the $T_{g}$ of the polymer, the fabricated 
organic micro-dynamic mechanical analyzers enable one to quantitatively determine the variation of storage modulus $\left(E^{\prime}\right)$ and the loss tangent $(\tan \delta)$ as a function of temperature for multiple organic material used as substrate in the MEMS structure as illustrated in Figure 4 for PET. Note that the devices showed excellent reversibility and repeatability in the thermo-mechanical characterization of organic materials for measurements at temperatures below the $T_{g}$ of the material to be characterized. This is confirmed by data in Figure $2 b$. Nevertheless, once the temperature reached and exceeded the $T_{g}$ of the polymer to be characterized, the devices' responses were not repeatable due to irreversible re-structurations of the material in its rubbery state.

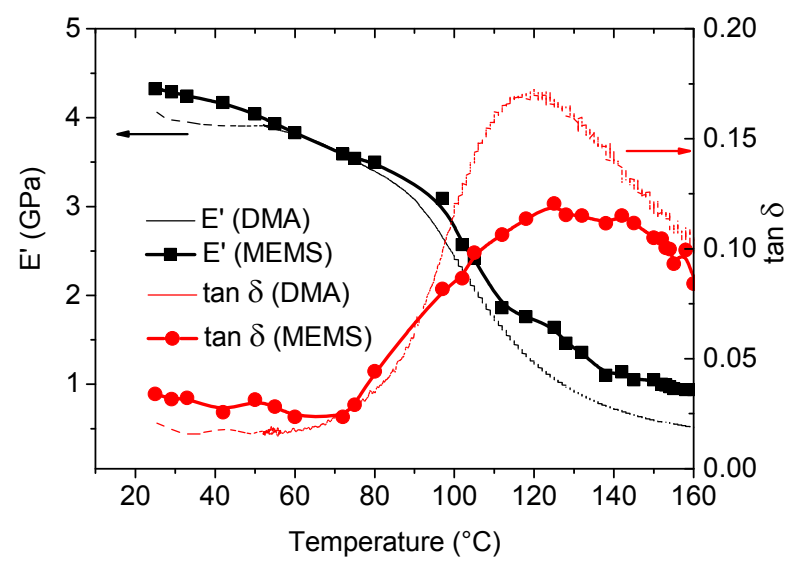

Figure 4 Mechanical properties of the PET-based U-shaped cantilever material as a function of temperature measured with the organic MEMS device and DMA

\section{Analytical modelling}

The Young's modulus of the material constituting the resonator has been determined by measuring the resonant frequency of the MEMS devices. Then, the quality factor used for the calculation of the $\tan \delta$ using the proposed MEMS has been extracted using the fitting model. ${ }^{25}$ The potential of the proposed characterization technique is indicated by the good correlation of the storage modulus and $\tan \delta$ values as measured with DMA. The slight differences between the DMA and MEMS measurements are due to the fact that $T_{g}$ has a kinetic component and is strongly influenced by the frequency (rate) of deformation. While the DMA measurements have been performed at a frequency rate of $1 \mathrm{~Hz}$, the MEMS measurements have been taken in the range of a few $\mathrm{kHz}$. As the frequency of the deformation increases, the molecular relaxations can only occur at higher temperatures and, as a consequence, $T_{g}$ increases with increasing frequency. In addition, the shape and magnitude of the $\tan \delta$ peak as well as the slope of the storage modulus in the transition region can also be affected. The tan $\delta$ of PET has been found to be 0.030 and 0.044 for DMA and MEMS resonator measurements at $20^{\circ} \mathrm{C}$, respectively. Similar measurements on PEN and paper substrates have been performed up to $50^{\circ} \mathrm{C}$ showing consistent results with DMA measurements. As a result, the proposed technique could be of great interest in the field of MEMS sensors where the performance of the devices are strongly influenced by any changes of mechanical properties of the materials due to temperature and/or frequency.

An approach has also been developed for determining the effective Young's modulus $E_{\text {eff }}$ (or effective storage modulus
$E_{\text {eff }}$ ) for "bimorph materials" (two-layer structures) obtained using the proposed organic MEMS device. The method is based on the following analytical model, ${ }^{26}$ which accounts for the non-uniform mass associated with the U-shaped cantilever. The major advantages of the analytical model proposed here is that the solution form is (1) based on a simple, yet rigorous, modification of the uniform cantilever case and (2) applicable to general end mass distributions. In the model normalized tip mass parameters $\left(\mu\right.$ and $\left.\mu_{e f f}\right)$ are utilized in the calculation of the resonant frequency in order to incorporate the effects of the translational and rotational inertia of the end portion as well as its eccentricity with respect to the ends of the legs:

$$
f_{r}=\frac{1}{2 \pi} \sqrt{\frac{3 E_{\text {eff }} I}{\bar{m} L^{4}\left(\mu_{\text {eff }}+\frac{33}{140}\right)}}
$$

where

$$
\begin{gathered}
\mu_{\text {eff }}=\mu\left[1+\frac{3}{2}\left(\frac{L_{0}}{L}\right)+\frac{3}{4}\left(\frac{L_{0}}{L}\right)^{2}+\frac{3}{16}\left(\frac{h}{L}\right)^{2}\right] \\
\mu \equiv \frac{\text { head mass }}{\text { total beam mass }}=\frac{\rho_{2} b_{0} h_{2} L_{0}+\rho_{1} b_{0} h_{1} L_{0}}{\rho_{2} b h_{2} L+\rho_{1} b h_{1} L}=\frac{b_{0} L_{0}}{b L},
\end{gathered}
$$

and

$$
\bar{m}=\rho_{1} b h_{1}+\rho_{2} b h_{2} .
$$

Since the Young's moduli of both materials are of the same order of magnitude, one may treat the flexural rigidity of the legs as being the same as that of a single layer of the same thickness and total width and having an effective modulus $E_{\text {eff. }}$ Thus, we take $I=\frac{b\left(h_{1}+h_{2}\right)^{3}}{12}$, with $b, L, b_{0}=1600 \mu \mathrm{m}$ and $L_{0}=$ $400 \mu \mathrm{m}$ being the width and length of the arms and end portion of the U-shaped cantilever beam, respectively, as shown in Figure 1c. Parameters $h_{1}, h_{2}, \rho_{1}$ and $\rho_{2}$ denote the thicknesses and mass densities of the substrate and nanocomposite layers, respectively. Measured values of resonant frequency used in conjunction with the above analytical expression permit one to easily back-calculate the effective modulus in the case of $100 \mu \mathrm{m}$ thick PET, $50 \mu \mathrm{m}$ thick PEN (PolyEthylene Naphtalate) and $200 \mu \mathrm{m}$ thick Paper (Powercoat ${ }^{\mathbb{B}}$ ) substrates coated with the nanocomposite. The values determined by this method have been compared with those from DMA measurements and show excellent agreement (Table 1).

\begin{tabular}{|c|c|c|c|c|}
\hline \multicolumn{2}{|c|}{ CNT-SU/8 on } & PET & PEN & Paper \\
\hline \multirow{2}{*}{$E_{\text {eff }}(\mathrm{GPa})$} & DMA & 4.43 & 2.90 & 4.33 \\
\cline { 2 - 5 } & MEMS & 4.38 & 2.81 & 4.11 \\
\hline \multirow{2}{*}{$E_{\text {eff }}{ }^{\prime}(\mathrm{MPa})$} & DMA & 131 & 290 & 625 \\
\cline { 2 - 5 } & MEMS & 192 & 256 & 471 \\
\hline \multirow{2}{*}{$\tan \delta$} & DMA & 0.030 & 0.100 & 0.144 \\
\cline { 2 - 5 } & MEMS & 0.044 & 0.091 & 0.115 \\
\hline
\end{tabular}

Table 1 Measured effective storage modulus ( $\left.E_{\text {eff' }}\right)$, loss modulus ( $E_{\text {eff }}$ ") and tan $\delta$ at room temperature of bimorph 
materials of CNT-SU/8 nanocomposite deposited on PET, PEN and paper substrates.

\section{Conclusions}

Organic U-shaped cantilever resonators can be fabricated using a quick, low-cost and environmentally friendly fabrication process. The developed fabrication method can be applied to a large panel of organic materials opening new routes toward the development of low-cost organic electronics devices. The fabricated devices have been tested optically and electrically. These responses have also been compared to FEM simulations. The high level of correlation between experiments and modeling confirm the achievement of the first piezoresistive transduction in organic MEMS resonators. The present MEMS exhibit an extreme sensitivity to temperature that compares well with that of inorganic materials. In spite of their low-cost, the present materials can therefore serve as viable candidates for various sensing applications. The sensitivity of the bimorph PET-CNT-SU/8 device with respect to temperature has been determined to be $-0.33 \% /{ }^{\circ} \mathrm{C}$ in the range of temperature $20-50^{\circ} \mathrm{C}$ together with a limit of detection of $0.112^{\circ} \mathrm{C}$, which is comparable to the best commercial Pt100 Class AA temperature sensors. As an example of another application, the present MEMS resonators have been used as microdynamic mechanical analyzers. Mechanical properties such as storage modulus and loss tangent of organic materials can be accurately extracted by recording the changes of resonant frequency and quality factor of the fabricated MEMS. Considering the cost, simplicity of fabrication, versatility and performance, the proposed MEMS offer exciting new possibilities across a wide spectrum of applications, including microsensors, mechanical energy harvesters, and microactuators.

\section{Acknowledgements}

This research was carried out within the framework of the LabEx AMADEuS, ANR-10-LABX-0042-AMADEUS, and was supported by a state grant handled by the National Agency of Research as part of the program Excellence Initiative IdEx Bordeaux, Grant N ANR-10-IDEX-0003-02.

\section{Notes and references}

${ }^{a}$ Université de Bordeaux, IMS, UMR5218, 351 cours de la Libération, 33405 Talence, France.

${ }^{b}$ Université de Bordeaux, CNRS, Centre de Recherche Paul Pascal, 115 Avenue Schweitzer, 33600 Pessac, France

c Department of Civil, Construction and Environmental Engineering, Marquette University, Milwaukee, WI, USA

\section{C. Liu, Adv. Mater. 2007, 19, 3783}

2 S. Sugiyama, S. Amaya and D. Viet Dao, Adv. Nat. Sci.: Nanosci. Nanotechnol. 2012, 3, 015009

$3 \mathrm{http}: / /$ www.promattex.com/plotter-traceur-decoupe/graphtec/graphtecce5000-40-craft-robo.php

4 C. Gau, H. S. Ko and H. T. Chen, Nanotechnology, 2009, 20, 185503
5 V. Seena, A. Fernandes, P. Pant, S. Mukherji and V. Ramgopal Rao, Nanotechnology, 2011, 22, 295501

6 D. Thuau, C. Ayela, P. Poulin and I. Dufour, Sensors and Actuators A: Physical, 2014, 209, 161

7 Alamusi, N. Hu, H. Fukunaga, S. Atobe, Y. L. Liu and J. H. Li, Sensors, 2011, 11, 10691

8 C. Stampfer, A. Jungen, R. Linderman, D. Obergfell, S. Roth and C. Hierold, Nano Lett., 2006, 6, 1449

9 A. Ferreira, P. Cardoso, D. Klosterman, J. A. Covas, F. W. J. van Hattum, F. Vaz and S. Lanceros-Mendez, Smart Mater. Struct., 2012, 21, 075008

10 M. Lillemose, L. Gammelgaard, J. Richter, E. V. Thomsen and A. Boisen, Composites Science and Technology, 2008, 68, 1831

11 A. Boisen, S. Dohn, S. Keller, S. Schmid, and M. Tenje, Rep. Prog. Phys,. 2011, 74, 036101

12 B. Hu, N. Hu, Y. Li, K. Akagi, W. Yuan, T. Watanabe and Y. Cai, Nanoscale Res. Lett. 2012, 7, 402

13 S. Xu, O. Rezvanian and M. A. Zikry, Smart Mater. Struct., 2013, 22, 055032

14 Z. Wang and X. Ye, Nanotechnology., 2013, 24, 265704

15 Y. H. Lin, M. E. McConney, M. C. LeMieux, S. Peleshanko, C. Jiang and S. Singamaneni, Adv. Mater., 2006, 18, 1157

16 M. C. LeMieux, M. E. McConney, Y. H. Lin, S. Singamaneni, H. Jiang and T. J. Bunning, Nano Lett., 2006, 6, 730

17 T. Larsen, S. Keller, S. Schmid, S. Dohn and A. Boisen, Microelectron. Eng., 2011, 88, 2311

18 Y. Shang, X. Ye, J. Feng, H. Zhou and Y. Wang, IEEE Sensors Journal, 2014, 14, 1304

19 S. Luo and T. Liu, Adv. Mater. 2013, 25, 5650

20 V. Skakalova, A. B. Kaiser, Y. S. Woo and S. Roth, Phys. Rev. B. 2006, 74, 085403

21 A. B. Kaiser, V. Skakalova and S. Roth, Physica E. 2008, 40, 2311

22 S. Bose, S. Schmid, T. Larsen, S. Keller, P. Sommer-Larsen, A. Boisen and K. Almdal, ACS Macro Lett. 2014, 3, 55

23 P. Colombi, P. Bergese, E. Bontempi, L. Borgese, S. Federici, S. Keller, A. Boisen and L. Depero, Meas. Sci. Technol. 2013, 24, 125603

24 I. Stachiv, D. Vokoun and Y. R. Jeng, App. Phys. Lett. 2014, 104, 083102

25 A. O. Niedermayer, T. Voglhuber-Brunnmaier, J. Sell and B. Jakoby, Meas. Sci. Technol. 2012, 23, 085107

26 S. Heinrich and I. Dufour, Proceedings of the $11^{\text {th }}$ Nanomechanical Sensing Workshop NMC 2014, April 30 - May 2 2014, Madrid, Spain, 2 pp. 